\title{
Direct measurement of the electronic spin diffusion length in a fully functional organic spin valve by low-energy muon spin rotation
}

\author{
A. J. Drew ${ }^{1,2 \star}$, J. Hoppler ${ }^{1,3}$, L. Schulz ${ }^{1}$, F. L. Pratt ${ }^{4}$, P. Desai ${ }^{2}$, P. Shakya ${ }^{2}$, T. Kreouzis ${ }^{2}$, W. P. Gillin ${ }^{2}$, \\ A. Suter ${ }^{5}$, N. A. Morley ${ }^{6}$, V. K. Malik ${ }^{1}$, A. Dubroka ${ }^{1}$, K. W. Kim ${ }^{1}$, H. Bouyanfif ${ }^{1}$, F. Bourquii', \\ C. Bernhard' ${ }^{1}$ R. Scheuermann ${ }^{5}$, G. J. Nieuwenhuys ${ }^{5}$, T. Prokscha ${ }^{5}$ and E. Morenzoni ${ }^{5}$
}

\begin{abstract}
Electronic devices that use the spin degree of freedom hold unique prospects for future technology. The performance of these 'spintronic' devices relies heavily on the efficient transfer of spin polarization across different layers and interfaces. This complex transfer process depends on individual material properties and also, most importantly, on the structural and electronic properties of the interfaces between the different materials and defects that are common to real devices. Knowledge of these factors is especially important for the relatively new field of organic spintronics, where there is a severe lack of suitable experimental techniques that can yield depth-resolved information about the spin polarization of charge carriers within buried layers of real devices. Here, we present a new depth-resolved technique for measuring the spin polarization of current-injected electrons in an organic spin valve and find the temperature dependence of the measured spin diffusion length is correlated with the device magnetoresistance.
\end{abstract}

$R$ ecently great efforts have been undertaken to use the spin degree of freedom in electronic devices. These activities are fuelled by the potential prospects of spin-electronic (or 'spintronic') devices for example in terms of increased processing speed and integration, non-volatility, reduced power consumption, multifunctionality and their suitability for quantum computing ${ }^{1}$. The most common method for using the spin in devices is based on the alignment of the electron spin ('up' or 'down') relative to either a reference magnetic field or the magnetization orientation of a ferromagnetic layer. Device operation normally proceeds with measuring a quantity such as the electrical current that depends on how the degree of spin alignment is transferred across the device. The so-called 'spin valve' is a prominent example of such a spin-enabled device that has already revolutionized hard-drive read heads and magnetic memory ${ }^{1}$. The efficient transfer of spin polarization in real device structures remains one of the most difficult challenges in spintronics, because it is dependent on more than just the properties of the individual materials that comprise the device.

Recently ${ }^{2,3}$, the use of organic materials in spintronics has become of significant interest, primarily owing to their ease and small cost of processing and electronic and structural flexibility. Furthermore, the extremely long spin coherence times found in organic materials offer considerable advantages over other materials ${ }^{3}$. This favourable property is related to two factors, first the weak spin-orbit coupling of light elements such as carbon and second to the small nuclear hyperfine interaction ${ }^{4,5}$. The latter arises because the electron transport in $\pi$-conjugated molecules is normally confined to molecular states, delocalized to the carbon rings, the predominant isotope of which, ${ }^{12} \mathrm{C}$, has zero nuclear $\operatorname{spin}^{4}$.

A common way to measure spin diffusion is based on time-resolved optical techniques, where spin-polarized charge carriers are created by the absorption of short pulses of circularly polarized light while their temporal and spatial evolution is probed via the absorption or reflection of suitable time-delayed pulses ${ }^{6,7}$. These optical pump/probe techniques generally enable studies of the spin diffusion in single materials or on the surface layers of devices. To the best of our knowledge, they have not been successfully applied to investigate the spin transport in buried layers of operational devices. Furthermore, they rely on a strong spin-orbit interaction, which is relatively weak in organic materials, meaning alternative techniques need to be developed to study spin coherence in organic materials.

Tunnelling-induced luminescence microscopy is another example of a technique that has revealed the characteristics of the injection of spin-polarized electrons, using a ferromagnetic metallic tip of a scanning tunnelling microscope to inject into a non-ferromagnetic semiconductor ${ }^{8}$. However, this is a surface-sensitive technique that cannot probe the polarization of injected electrons from a buried interface in an operational device.

Recently it has been shown that it is possible to measure spin injection from an in-plane magnetized ferromagnetic film into a semiconductor ${ }^{9}$, even below the ferromagnetic contacts, using the magneto-optical Kerr effect. This was made possible by carrying out the measurement on a cleaved edge of the sample, but this is not

\footnotetext{
${ }^{1}$ Department of Physics and Fribourg Center for Nanomaterials, University of Fribourg, Chemin du Musée 3, CH-1700 Fribourg, Switzerland, ${ }^{2}$ Department of Physics, Queen Mary, University of London, Mile End Road, London, E1 4NS, UK, ${ }^{3}$ Laboratory for Neutron Scattering, Paul Scherrer Institute, CH-5232 Villigen PSI, Switzerland, ${ }^{4}$ ISIS Pulsed Neutron and Muon Source, Rutherford Appleton Laboratory, Chilton, Didcot, OX11 0QX, UK, ${ }^{5}$ Laboratory for Muon-Spin Spectroscopy, Paul Scherrer Institute, CH-5232 Villigen PSI, Switzerland, ${ }^{6}$ Department of Engineering Materials, University of Sheffield, Mappin Street, Sheffield, S1 3JD, UK. *e-mail: A.J.Drew@qmul.ac.uk.
} 
a

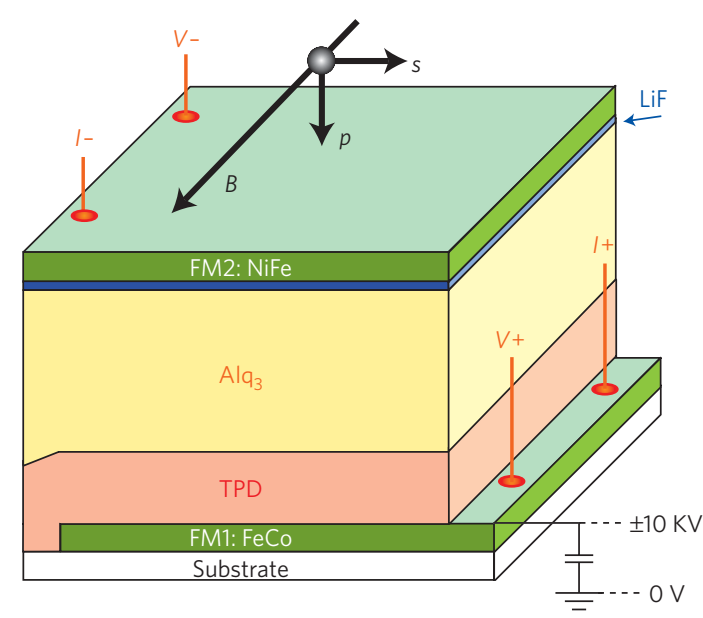

b

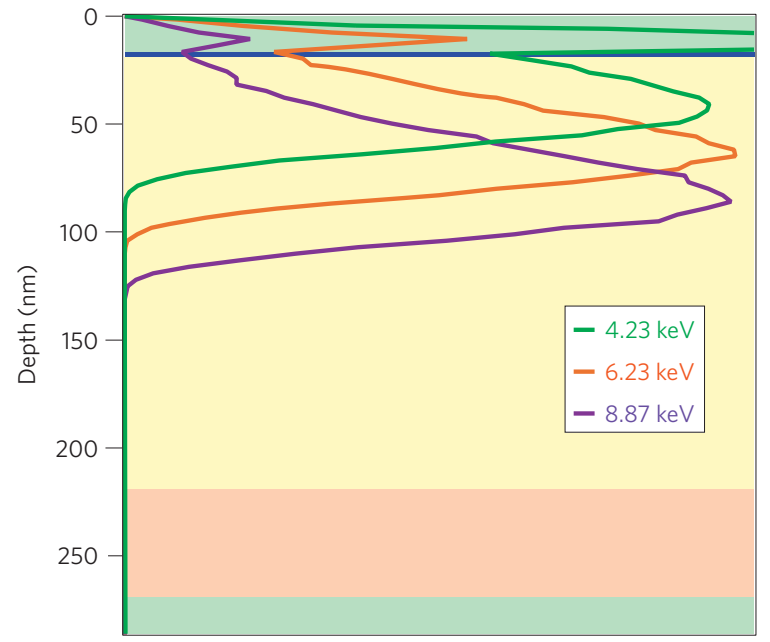

Figure 1 | Schematic diagram of the experimental set-up. a, A schematic diagram showing the structure of our device. FM1 (FM2): ferromagnetic layer 1 (2). b, Depth profile showing the calculated probability that a positively charged muon with an implantation energy of $4.23,6.23$ or $8.87 \mathrm{keV}$ (green, orange or purple line) comes to rest at a certain depth within the device (so-called stopping or implantation profile).

compatible with devices based on organic materials because they are not easily cleaved.

The only practical way of accessing the spin diffusion in organic spin devices is by measuring the magnetoresistance $e^{2,3,10-12}$ or by tunnelling into a superconductor ${ }^{13}$. The former technique does not distinguish between the different sources of spin decoherence in these complex devices whereas the latter provides this information only at a particular interface. In both cases, the extraction of a spin diffusion length requires measurements on a series of devices of different dimensions but with otherwise identical properties. Typical values of the spin diffusion length in organic materials are 5-40 nm in tris-(8-hydroxyquinoline) aluminium ${ }^{2,3}\left(\mathrm{Alq}_{3}\right), 130 \mathrm{~nm}$ in carbon nanotubes ${ }^{11}$ and $200 \mathrm{~nm}$ in polymers ${ }^{12}$. However, there are important issues that still need to be resolved regarding how the precise growth parameters influence the spin transport in organic materials, which exhibits a large variation. For example, contrary to the results presented here and in the literature $e^{2,3,14}$, the absence of spin transport has recently been reported in spin valves based on $\mathrm{Alq}_{3}$ (ref. 15). The apparent absence of a correlation between the growth conditions and device performance demonstrates the necessity of carrying out spatially resolved measurements of spin injection in a single realistic spin-enabled device.

\section{Measurement of spin penetration}

We report here what is, to the best of our knowledge, the first direct and depth-resolved measurement of spin penetration away from an injection interface in a realistic and fully functional organic spin valve. For this, we used the technique of low-energy muon spin rotation ${ }^{16,17}$ (LE- $\mu$ SR), which has previously been applied successfully to obtain the depth-resolved profile of the local magnetization in various thin films and heterostructures ${ }^{18,19}$. The stopping distribution of the fully spin-polarized muons can be varied here on the scale of about 3-200 nm through the control of the muon implantation energy. The obtained $\mu$ SR spectra yield the probability distribution of the local magnetic field at the muon sites, often referred to as a ' $\mu$ SR lineshape', which contains direct information on the local spin polarization of the charge carriers.

A schematic diagram of the investigated spin-valve structure is shown in Fig. 1a. The device has an active area of $18 \times 18 \mathrm{~mm}$, comprising NiFe $17 \mathrm{~nm} / \mathrm{LiF} 1.9 \mathrm{~nm} / \mathrm{Alq}_{3} 200 \mathrm{~nm} / \mathrm{TPD} 50 \mathrm{~nm} / \mathrm{FeCo}$ $17 \mathrm{~nm}$ with Au contact pads at the edges (TPD: $N, N^{\prime}$-diphenyl$N$ - $N^{\prime}$-bis(3-methylphenyl)-1, 1'-biphenyl-4, 4'-di amine). We injected spin-polarized electrons from the top $\mathrm{NiFe}$ electrode through a $\mathrm{LiF}$ tunnel barrier ${ }^{20}$ into $\mathrm{Alq}_{3}$, in which the muons were implanted. The lowest two layers of our device were the hole injection layer TPD (ref. 21) and the bottom electrode FeCo. The magnetic field was applied parallel to the layers and perpendicular to the muon's initial spin direction and momentum. We used a bespoke floating power supply/volt meter that could bias the sample to a high degree of accuracy $( \pm 0.1 \mathrm{nA}$ and $\pm 0.1 \mu \mathrm{V})$ while floating the sample at $\pm 10 \mathrm{kV}$. The high voltage is necessary to control the muon implantation energy and thus the muon stopping distribution within the device. This is indicated in Fig. $1 \mathrm{~b}$ for the three muon implantation energies we used. This configuration enabled us to measure the induced magnetization due to the spin-polarized charge carriers that are injected from the ferromagnetic layer while simultaneously carrying out in situ magnetoresistance measurements.

\section{Proof of principle}

Figure 2a shows the distribution of local magnetization in the sample, where the muons are stopped well inside the organic $\mathrm{Alq}_{3}$ layer, at an implantation energy of $6.23 \mathrm{keV}$ at $T=90 \mathrm{~K}$. The measurements proceeded by first applying a field of $100 \mathrm{mT}$ to ensure that the ferromagnetic layers were saturated, after which the magnetic field was reduced to $29 \mathrm{mT}$. The $\mu \mathrm{SR}$ spectra were first obtained with the current on and then with the current off.

Initially we discuss the $\mu S R$ lineshape in zero current. As shown in Fig. 2a, it has a characteristic shape with a pronounced tail towards the high-field side. We express this asymmetry of the lineshape in terms of the so-called skewness parameter, $\Delta$, as defined in Supplementary Information. The present case of a lineshape with an asymmetry towards the high-field side is denoted as skewed to the 'right', or positively skewed. We note that this zero-current lineshape can be explained in terms of the dipolar stray fields that originate from the roughness of the interface between the ferromagnetic and the spacer layers ${ }^{22,23}$. We have carried out model calculations of how these dipolar fields extend into the organic layer using as input parameters the ferromagnetic moment and the interface roughness as extracted from magnetometry, atomic force microscopy, X-ray and neutron reflectivity measurements, which are contained in Supplementary Information. Our calculations as shown by the red solid line in Fig. 2a are in very good agreement with the data represented by the red circles.

More importantly, however, on applying an electric current through our device and thus injecting spin-polarized carriers from the ferromagnetic top layer into the buried organic layer, small but significant changes are observed in the lineshape (Fig. 2a, blue symbols). These are further detailed in the inset of Fig. 2a, which 

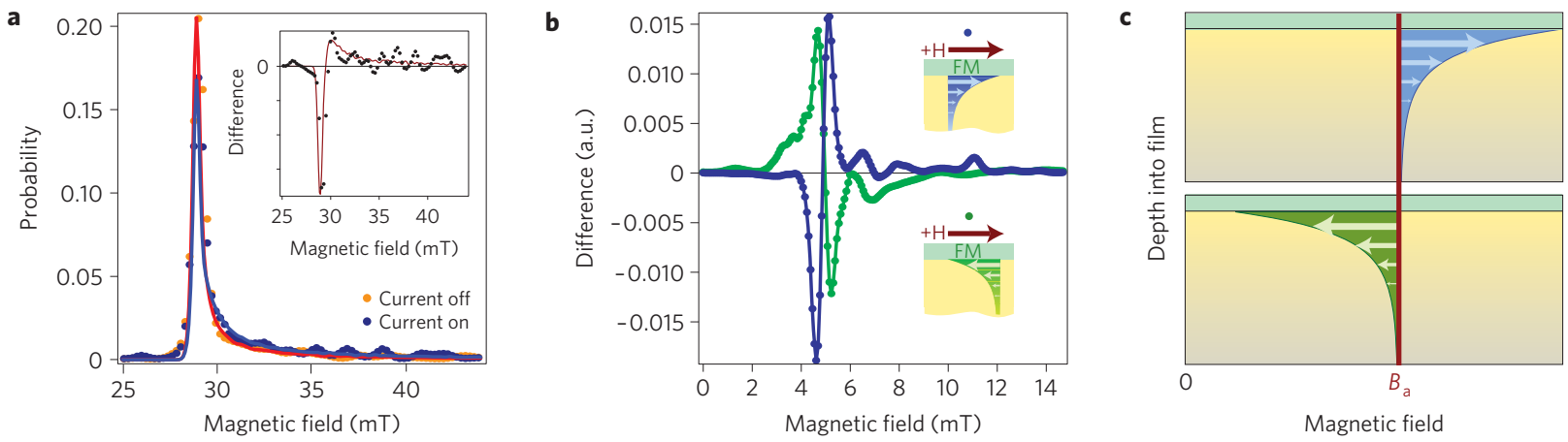

Figure 2 | Influence of spin injection on the $\boldsymbol{\mu S R}$ spectra. a, The distribution of magnetic fields for the implantation energy of $6.23 \mathrm{keV}$ (for which the muon stopping distribution is shown in Fig. $1 \mathrm{~b}$ by the orange line) and an applied magnetic field of $29 \mathrm{mT}$. The data with a current density of 0 and $3 \mathrm{~mA} \mathrm{~cm}^{-2}$ are shown in red and blue, respectively. The circles show the experimentally measured data that exhibit a small but significant difference between current-on and -off. Solid lines show the result of model calculations for a dipolar field distribution due to rough interfaces and due to the current-induced injection of spin-polarized charge carriers where the corresponding parameters were extracted from the time-domain fits (as discussed in the text). Inset: The difference between the two data sets (black circles) and the difference predicted by the model (red line). The small oscillation that can be observed in the model is a due to the model's finite size. $\mathbf{b}$. The difference between the experimentally measured distribution of magnetic fields with the injection current on and off, for an applied field of $5 \mathrm{mT}$. The blue and green lines show different configurations, where the direction of the external field with respect to the spin polarization of the injected charge carriers is either parallel or antiparallel. It is clear that when in the parallel configuration, the current-on lineshape is skewed to higher magnetic fields, whereas in the antiparallel configuration, the current-on lineshape is skewed to lower magnetic fields. c, Schematic diagram of the two cases shown in b, showing the expected spatial distributions of local magnetic field in the organic layer for both configurations. When the spins of the injected charge carriers are aligned (anti-aligned) with respect to the applied field, the $\mu$ SR lineshape is skewed to the higher (lower) fields.

shows the difference between the current-on and -off spectra. Specifically, in the current-on condition, the muons experience a noticeable enhancement of the local magnetic fields, which gives rise to an increase in the positive skewness of the $\mu \mathrm{SR}$ lineshape. This is the exact effect that is to be expected if spin-polarized charge carriers are injected into the organic layer, because they would produce a local magnetization that adds to the dipolar stray fields from the ferromagnetic layers. We note that the observed effect is unlikely to be due to sample heating, which is insignificant for a current density of only $3 \mathrm{~mA} \mathrm{~cm}^{-2}$ and which should not affect the skewness of the $\mu S R$ lineshape. Similar arguments apply for a possible effect from the injection of unpolarized electrons.

To ensure that the observed changes in the $\mu \mathrm{SR}$ lineshape are indeed brought about by the injection of spin-polarized charge carriers, we carried out a set of similar measurements where we reversed the direction of the injected spins from the top ferromagnetic electrode with respect to the external magnetic field. This was achieved by first saturating the magnetizations of both of the ferromagnetic layers in a field of $100 \mathrm{mT}$ and then reducing the applied field to $5 \mathrm{mT}$, before carrying out the first $\mu \mathrm{SR}$ current-on/off measurement. We then applied a field of $-100 \mathrm{mT}$ to saturate the magnetization of the ferromagnetic layers in the reverse direction before returning the field to $5 \mathrm{mT}$ and collecting the second set of $\mu$ SR spectra. These two configurations are shown in the inset of Fig. $2 b$.

For the latter case, the spin polarization of the injected electrons remains predominantly antiparallel with respect to the direction of the external magnetic field. This is clearly demonstrated by the magnetoresistance measurements shown in Fig. 3a. We have plotted the difference between the current-on and -off spectra in Fig. $2 \mathrm{~b}$ for the two different configurations, where it can be seen that there is a shift in weight from low to high magnetic fields for the parallel orientation of the external magnetic field and the injected spin. For the antiparallel orientation, the shift is opposite-from high to low magnetic fields. This phenomenon can easily be understood by consulting Fig. 2c, where the external field (red) and the current-induced magnetization inside the organic layer (blue and green) are shown in the schematic diagrams for both configurations. When the spin is injected parallel to the magnetic field, the net contribution results in a positive skewness. However, when the spin is injected antiparallel to the magnetic field, the spin component is subtracted, resulting in a negative skewness. These measurements preclude any explanation in terms of intrinsic organic magnetoresistance and therefore confirm the scenario that the current-induced changes in the local magnetic field distribution are brought about by the injection of spin-polarized charge carriers.

\section{Quantitative analysis}

We now present a quantitative analysis in terms of the skewness parameter, $\Delta$, based on fits to the time-domain $\mu S R$ spectra (as outlined in Supplementary Information). Different from the Fourier-transformation analysis presented above, which is most suitable to visualize changes in the current-induced magnetization, this analysis gives more direct and quantitative information about the relevant parameters and gives an overview of the measurements taken of the sample in different states. Figure 3 shows the result of this quantitative analysis for a series of five measurements carried out at different points during a cyclic change of the external magnetic field. Figure 3a shows the corresponding magnetoresistance hysteresis loop, where the conditions for the different $\mu$ SR measurements are marked with numbers (the $\mu$ SR lineshapes for the data at points 1,2 and 5 were already shown in Fig. 2a,b). The corresponding orientation of the magnetization in the ferromagnetic layers and the external magnetic field is shown on the right-hand side of Fig. 3. Figure $3 \mathrm{~b}$ shows the change in the obtained skewness parameter at points $1-5$ for the current-off and current-on conditions. In the first place, we note that the changes of $\Delta$ shown in Fig. $3 \mathrm{~b}$ are indeed consistent with the scenario of a current-induced injection of spin-polarized charge carriers into the organic layer, as the direction of the shift in $\Delta$ is determined by the relative orientation of the injected spin polarization with respect to the external field. It is furthermore evident from Fig. $3 \mathrm{~b}$ that the magnitude of the shifts in $\Delta$ significantly exceeds the error bars that are derived from the Poissonian statistics. Taking into account the full body of evidence 
presented so far, we therefore conclude that our work presents, to the best of our knowledge, the first direct measurement of the spin coherence of current-injected electrons within a realistic organic spin-valve device.

Using some reasonable assumptions, we can also extract from our data estimates for the spin diffusion length. For the sake of a straightforward implementation in the fitting routine, we assume that the average dipolar magnetic flux density in the organic layer decays exponentially away from the interfaces. This assumption is supported by literature ${ }^{22,23}$ and justified by our numerical calculations (see Supplementary Information). The total flux density thus takes the following form:

$$
\begin{aligned}
B(z)= & B_{\mathrm{d} 1} \exp \left(-z / \lambda_{\mathrm{d} 1}\right)+B_{\mathrm{d} 2} \exp \left(-[d-z] / \lambda_{\mathrm{d} 2}\right) \\
& +B_{\mathrm{s}} \exp \left(-z / \lambda_{\mathrm{s}}\right)+B_{\mathrm{a}},
\end{aligned}
$$

where $B_{\mathrm{a}}$ is the applied magnetic field, $B_{\mathrm{d} 1}$ and $\lambda_{\mathrm{d} 1}\left(B_{\mathrm{d} 2}\right.$ and $\left.\lambda_{\mathrm{d} 2}\right)$ are the magnitude and the characteristic length scale of the average dipolar field at the top (bottom) interface, $d$ is the thickness of the organic spacer layer and $B_{\mathrm{s}}$ and $\lambda_{\mathrm{s}}$ are the magnitude and decay length of the magnetization due to the injected spin-polarized carriers. We carried out the fits simultaneously to the data at different implantation energies by weighting them with the muon stopping profiles as shown in Fig. 1b. We determined the dipolar contribution to the $\mu$ SR lineshape from the current-off data and kept it unchanged for fitting the current-on data. This procedure relies on the second reasonable assumption that the domain structure in the ferromagnetic layer and thus the dipolar stray fields are not affected by the small current flow. The obtained parameters for the spin polarization of the injected carriers are $\lambda_{\mathrm{s}} \approx 10 \pm 1 \mathrm{~nm}$ and $B_{\mathrm{s}} \approx 200 \pm 20 \mathrm{mT}$ at $90 \mathrm{~K}$.

As mentioned above, we have also carried out numerical calculations of the dipolar contribution in the organic layer. The red line in Fig. 2a shows the calculated $\mu$ SR lineshape for the current-off situation. The blue line shows the corresponding current-on $\mu \mathrm{SR}$ lineshape with the extra contribution due to the spin-polarized charge carriers, determined from the time-domain fits explained in the previous paragraph. The inset details the difference between the current-on and the current-off situation. The good agreement between the calculated (lines) and the measured (symbols) data testifies for the consistency of our fitting procedure.

Figure 4 shows the obtained temperature dependence of the spin diffusion length as extracted from our $\mu S R$ data and compares it to the magnetoresistance measurements. The qualitative agreement between these microscopic and macroscopic techniques suggests that the spin diffusion length is a key parameter of spin transport in organic materials. This contrasts with the conclusion of recent work where the magnetoresistance observed in devices based on a variety of organic materials seemed to track the injection polarization $^{14}$, which is explained in terms of the temperature dependence of the surface polarization of the ferromagnetic $\mathrm{La}_{2 / 3} \mathrm{Sr}_{1 / 3} \mathrm{MnO}_{3}$ layer ${ }^{24}$. In our study, we avoided this by using a transition-metal ferromagnetic with a high Curie temperature for both of our injection electrodes, which ensures a weak variation of the spin injection efficiency in the experimental temperature range $^{25}$. It is therefore unlikely that the abrupt change in the magnetoresistance of our devices at around $40 \mathrm{~K}$ is caused by the ferromagnetic electrodes. However, direct measurements of the injection polarization could be obtained from future LE- $\mu S R$ measurements of the magnetization at the ferromagnetic interface. As to the origin of the step-like increase of the spin diffusion length below $40 \mathrm{~K}$, this could be either related to an increase in the charge-carrier mobility or a decrease in the spin relaxation rate. The former possibility is not supported by measurements
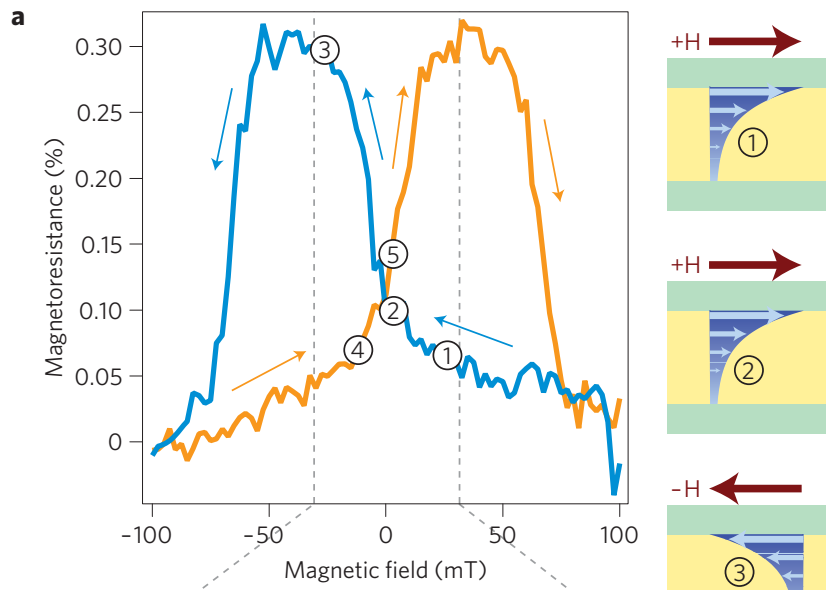

b

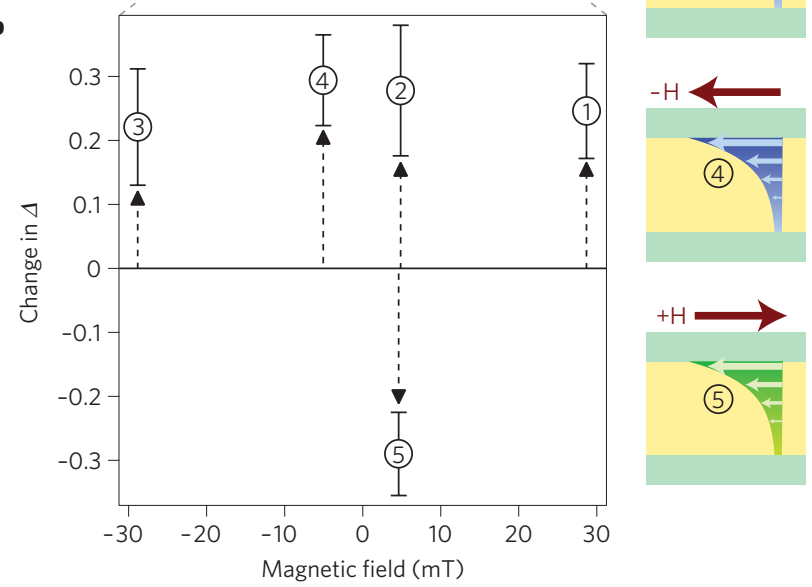

Figure 3 | Correlation between the direction of spin polarization of the injected carriers and the change in the $\boldsymbol{\mu S R}$ spectra. $\mathbf{a}$, Hysteresis loop of the magnetoresistance as a function of increasing (orange line) and decreasing (blue line) external magnetic field. Marked by numbers 1-5 are the points at which the $\mu$ SR lineshapes have been measured (for current-off and current-on). Schematic diagrams of the corresponding orientation of the magnetization of the ferromagnetic layers with respect to the external magnetic field are shown to the right. $\mathbf{b}$, Change of the skewness parameter obtained from the time-domain analysis of the muon measurements, where the measurement conditions are labelled as defined in $\mathbf{a}$. Especially important are points 3 and 4, which establish that the sign of the skewness is determined by the relative direction of the external magnetic fields and the polarization of the injected carriers. Error bars represent one standard deviation.

of the carrier mobility in $\mathrm{Alq}_{3}$ (refs 26,27). On the other hand, the observation of a marked increase at $40 \mathrm{~K}$ in the lifetime of the hole-electron triplet states in $\mathrm{Alq}_{3}$ (ref. 28) suggests there is a corresponding decrease of the spin relaxation rate. Furthermore, the electron paramagnetic resonance line intensity in $\mathrm{Alq}_{3}$ exhibits a similar step-like increase below $40 \mathrm{~K}$ (ref. 29). To the best of our knowledge, the mechanism that leads to this marked decrease of the spin relaxation rate below $40 \mathrm{~K}$ has not yet been identified. Our experiments highlight that it markedly affects the performance of organic spin-valve devices and thus should motivate efforts to better understand the microscopic spin relaxation mechanisms in organic materials. Finally, we note that the LE- $\mu$ SR data at low and at high temperature as well as the magnetoresistance data shown in Fig. 4 were obtained on different samples. Although the characteristic temperature dependence of the magnetoresistance has been well established, the existence of a corresponding unique trend in the $\mu$ SR data requires further data, 


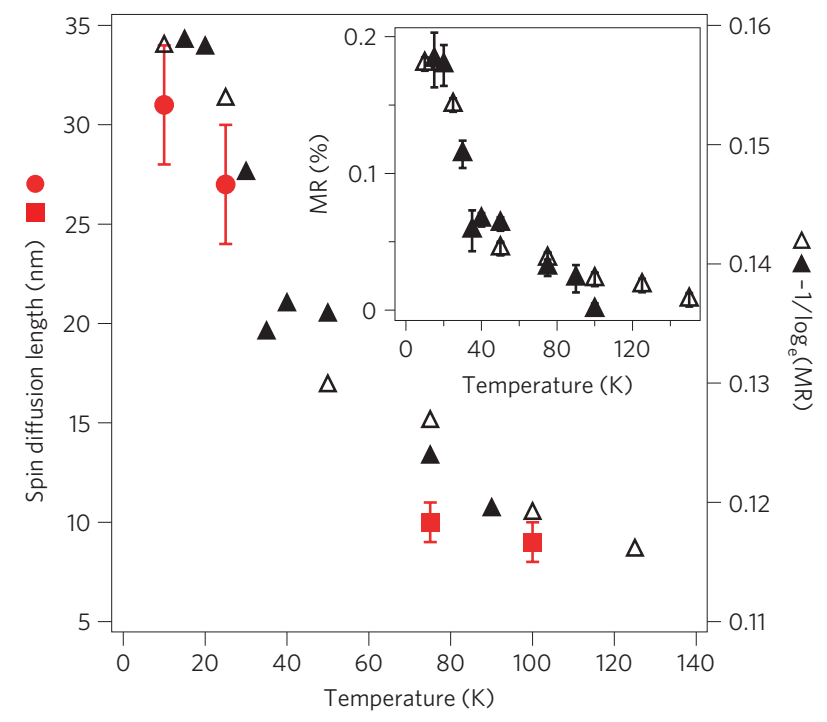

Figure 4 | Correlation between spin diffusion length and

magnetoresistance. The temperature dependence of the spin diffusion length extracted from the muon measurements (red, two different samples indicated by the squares and circles) plotted together with the temperature dependence of magnetoresistance (black symbols) for a further two different samples (indicated by the open and filled triangles), where there is clearly a qualitative agreement between the macroscopic and microscopic techniques. Inset: The same magnetoresistance data plotted on a linear scale, where an abrupt increase below $40 \mathrm{~K}$ is observed. Error bars represent one standard deviation for the spin diffusion length and are an estimate based on the scatter in the data for the magnetoresistance measurements.

that is, the full temperature dependence needs to be measured for a single spin-valve structure.

\section{Summary}

In summary, we have demonstrated that LE- $\mu$ SR measurements can provide unique depth-resolved information about the degree of spin polarization of the injected charge carriers within a buried active layer of a functional organic spin-valve device. Our results highlight the unique potential of the LE- $\mu$ SR technique to reveal the role of the various mechanisms that limit the spin coherence in fully functional and realistic devices, especially in those involving organic materials. Specifically, LE- $\mu$ SR can enable differentiation between bulk- and interface-related spin decoherence phenomena. As a first step, we have presented a comparison between microscopic $\mu$ SR and macroscopic magnetoresistance measurements, which suggest that the spin diffusion length is a key parameter for the spin transport in organic materials.

\section{Methods}

TPD and $\mathrm{Alq}_{3}$ (99.995\% pure) were purchased from Aldrich and purified by train-sublimation under a $10^{-6}$ mbar vacuum. The deposition of the organic layers was carried out using a Kurt J. Lesker SPECTROS evaporation system under $\sim 10^{-7}$ mbar. Magnetic layers were evaporated in a separate system under $\sim 10^{-6}$ mbar. A calibrated oscillating quartz-crystal monitor was used to determine the rate and thickness of the deposited layers. The deposition rate of the organic materials was maintained at $2 \AA \mathrm{s}^{-1}$ and the magnetic contacts varied from $\sim 1$ to $10 \AA \mathrm{s}^{-1}$. Shadow masks were used to define the device geometry.

To extend the scope of the $\mu$ SR technique to thin films, spin-polarized muon beams with tunable energies in the kilo-electronvolt range with a narrow energy distribution have been developed at the Paul Scherrer Institute. The aptly named LE- $\mu$ SR spectrometer has been successful in providing a beam of muons that can be routinely used for depth-dependent investigations on the nanometre scale ${ }^{30,31}$.

In our experiment, $110 \mathrm{~nm}$ of a weakly bound van der Waals cryosolid ( solid- $\mathrm{N}_{2}$ ) was deposited on the downstream side of a cold metal substrate, which moderates a fraction of an intense surface muon beam to $\sim 15 \mathrm{eV}$ (with a similar r.m.s. energy spread) while conserving the initial full polarization ${ }^{32}$. The epithermal muons are extracted (by applying up to $+20 \mathrm{kV}$ to the moderator substrate), transported and focused by electrostatic elements to the sample. A trigger detector provides a muon start signal by detecting secondary electrons, released by the muons when passing through a $2 \mu \mathrm{g} \mathrm{cm}^{-2}$ carbon foil onto a microchannel plate detector. The mean implantation energy can be varied between 0.5 and $30 \mathrm{keV}$ by choosing the appropriate moderator, transport and sample voltages. The muon's stopping profile has been calculated using a Monte Carlo algorithm TRIM.SP (ref. 33)

\section{References}

1. Wolf, S. A. et al. Spintronics: A spin-based electronics vision for the future. Science 294, 1488-1495 (2001)

2. Xiong, Z. H., Wu, D., Vardeny, Z. V. \& Shi, J. Giant magnetoresistance in organic spin-valves. Nature 427, 821-824 (2004).

3. Pramanik, S. et al. Observation of extremely long spin relaxation times in an organic nanowire spin valve. Nature Nano. 2, 216-219 (2007).

4. Sanvito, S. \& Rocha, A. R. Molecular-spintronics: The art of driving spin through molecules. J. Comput. Theor. Nanosci. 3, 624-642 (2006).

5. Khaetskii, A. \& Nazarov, Y. V. Spin-flip transitions between Zeeman sublevels in semiconductor quantum dots. Phys. Rev. B 63, 184433 (2001).

6. Cameron, A. R., Riblet, P. \& Miller, P. A. Spin gratings and the measurement of electron drift mobility in multiple quantum well semiconductors. Phys. Rev. Lett. 76, 4793-4796 (1996).

7. Kikkawa, J. M. \& Awschalom, D. D. Lateral drag of spin coherence in gallium arsenide. Nature 397, 139-141 (1999).

8. LaBella, V. et al. Spatially resolved spin-injection probability for gallium arsenide. Science 292, 1518-1521 (2001).

9. Kotissek, P. et al. Cross-sectional imaging of spin injection into a semiconductor. Nature Phys. 3, 872-877 (2007).

10. Julliere, M. Tunneling between ferromagnetic films. Phys. Lett. A 54, 225-226 (1975).

11. Tsukagoshi, K., Alphenaar, B. W. \& Ago, H. Coherent transport of electron spin in a ferromagnetically contacted carbon nanotube. Nature 401, 572-574 (1999).

12. Dediu, V. et al. Room temperature spin polarized injection in organic semiconductor. Solid State Commun. 122, 181-184 (2002).

13. Shim, J. H. et al. Large spin diffusion length in an amorphous organic semiconductor. Phys. Rev. Lett. 100, 226603 (2008).

14. Wang, F. J., Yang, C. G., Vardeny, Z. V. \& Li, X. G. Spin response in organic spin valves based on $\mathrm{La}_{2} / 3 \mathrm{Sr}_{1} / 3 \mathrm{MnO}_{3}$ electrodes. Phys. Rev. B 75, 245324 (2007).

15. Jiang, J. S., Pearson, J. E. \& Bader, S. D. Absence of spin transport in the organic semiconductor $\mathrm{Alq}_{3}$. Phys. Rev. B 77, 035303 (2008).

16. Prokscha, T. et al. The new high-intensity surface muon beam $\mu \mathrm{E} 4$ for the generation of low-energy muons at PSI. Physica B 374, 460-462 (2006).

17. Bakule, P. \& Morenzoni, E. Generation and applications of slow polarized muons. Contemp. Phys. 45, 203-225 (2004).

18. Drew, A. et al. Coexistence and coupling of superconductivity and magnetism in thin film structures. Phys. Rev. Lett. 95, 197201 (2005).

19. Suter, A. et al. Direct observation of nonlocal effects in a superconductor. Phys. Rev. Lett. 92, 087001 (2004).

20. Yunus, M. et al. Ambipolar electrical spin injection and spin transport in organic semiconductors. J. Appl. Phys. 103, 103714 (2008).

21. Tang, C. W. \& VanSlyke, S. A. Organic electroluminescent diodes. Appl. Phys. Lett. 51, 913-915 (1987).

22. Demonkritov, S. et al. Magnetic-dipole mechanism for biquadratic interlayer coupling. Phys. Rev. B 49, 720-723 (1994).

23. Altbir, D., Kiwi, M., Ramirez, R. \& Schuller, I. K. Dipolar interaction and its interplay with interface roughness. J. Magn. Magn. Mater. 149, L246-L250 (1995).

24. Park, J. H. et al. Magnetic properties at surface boundary of a half-metallic ferromagnet $\mathrm{La}_{0.7} \mathrm{Sr}_{0.3} \mathrm{MnO}_{3}$. Phys. Rev. Lett. 81, 1953-1956 (1998).

25. Tsymbal, E. Y., Mryasov, O. N. \& LeClair, P. R. Spin-dependent tunnelling in magnetic tunnel junctions. J. Phys. Condens. Matter 15, R109-142 (2003).

26. Malliaras, G. G, Shen, Y., Dunlap, D. H., Murata, H. \& Kafafi, Z. H. Nondispersive electron transport in $\mathrm{Alq}_{3}$. Appl. Phys. Lett. 79, 2582-2582 (2001).

27. Bässler, H. Charge transport in organic photoconductors. Phys. Status Solidi B 175, 15-56 (1993).

28. Cölle, M., Gärditz, C. \& Braun, M. The triplet state in tris-(8-hydroxyquinoline)aluminium. J. Appl. Phys. 96, 6133-6141 (2004).

29. Grecu, M. N., Mirea, A., Ghica, C., Cölle, M. \& Schwoerer, M. Paramagnetic defect centres in crystalline $\mathrm{Alq}_{3}$. J. Phys. Condens. Matter 17, 6271-6283 (2005). 
30. Prokscha, T. et al. The new $\mu \mathrm{E} 4$ beam at PSI: A hybrid-type large acceptance channel for the generation of a high intensity surface-muon beam. $\mathrm{Nucl}$. Instrum. Methods A 595, 317-331 (2008).

31. Prokscha, T. et al. <http://lmu.web.psi.ch/lem/index.html>.

32. Morenzoni, E. et al. Generation of very slow polarized positive muons. Phys. Rev. Lett. 72, 2793-2796 (1994).

33. Morenzoni, E. et al. Implantation studies of $\mathrm{keV}$ positive muons in thin metallic layers. Nucl. Instrum. Methods B 192, 254-266 (2002).

\section{Acknowledgements}

We acknowledge financial support from the Schweizer Nationalfonds (SNF) Nos 200021-111690 and 200020-119784 and the NCCR Materials with Novel Electronic Properties (MaNEP) programme. Experiments were carried out at the Swiss Muon Source, Paul Scherrer Institute, Villigen, Switzerland.

\section{Author contributions}

A.J.D. was responsible for project planning. A.J.D, J.H., L.S., F.L.P., A.S., N.A.M., V.K.M., A.D., K.W.K., H.B., R.S., G.J.N., T.P. and E.M. were responsible for the experimental measurements. A.J.D., J.H., L.S., F.L.P., A.S., T.K., W.P.G. and C.B. were responsible for the analysis and interpretation. A.J.D., P.D., P.S., T.K. and W.P.G. were responsible for the sample growth and characterization. The bespoke floating current source was designed, built and tested by A.J.D., A.S. and F.B. 